\title{
Helicobacter pylori may protect against IBD-a mechanistic insight
}

Helicobacter pylori DNA can suppress the release of proinflammatory cytokines by dendritic cells (DCs) and attenuate the severity of colitis in a mouse model of IBD, new findings show. "There are increasing data suggesting $H$. pylori protects against chronic inflammatory diseases, such as IBD," says John Kao, the corresponding author of the study. "The goal of this project was to attempt to define a mechanism for this clinical observation."

H. pylori DNA is detectable in the stool of infected individuals and contains a high proportion of immunoregulatory sequences, the researchers found. When they added $H$. pylori DNA to cultures of mouse bone-marrow-derived DCs and human plasmacytoid DCs, production of type I interferons (IFNs) decreased. Previous studies have implicated these proinflammatory IFNs in the pathogenesis of other inflammatory diseases.

Furthermore, mice that had been pretreated with $H$. pylori DNA before inducing colitis with dextran sodium sulfate experienced less severe symptoms (including reduced bleeding and weight loss) compared with control animals. The researchers also showed that serum levels of type I IFNs were lower in individuals with $H$. pylori infection $(n=22)$ than in those without $(n=24)$.

"Our hypothesis is that $H$. pylori DNA shed from the stomach into the distal intestinal system can suppress inflammation, which may decrease the likelihood of developing IBD," concludes Kao. He adds that the results have important implications for the debate on global vaccination against $H$. pylori.

Shreeya Nanda

Original article Luther, J. et al. Helicobacter pylori DNA decreases pro-inflammatory cytokine production by dendritic cells and attenuates dextran sodium sulphateinduced colitis. Gut doi:10.1136/gut.2010.220087 